\title{
THE PROPORTIONING OF ELECTRODES FOR
} FURNACES

\section{BY CARL HERING}

Introductory. The usual rules for proportioning electrodes for electric furnaces have been based on such factors as allowable current densities, least practicable resistance (hence shortness and large section), lowest heat conduction, the summation of losses due to the electric resistance and to the heat conduction to get the total, etc.

Believing that these laws were not based on correct principles, and were therefore unsatisfactory and perhaps even misleading, and as apparently no one had made a thorough investigation of this subject based on unquestioned fundamental laws, the writer some time ago made a careful study of the true principles underlying the proper proportioning of electrodes, based on indisputable physical laws. The results of this analysis showed that our former rules were not only entirely inadequate, but were in part quite incorrect and led to entirely wrong conclusions. And as the value of the annual loss of energy in such electrodes is very large, the matter of the correct proportioning is of considerable commercial importance as well as of interest to the engineer. This analytical investigation was then s'ipplemented with an experimental one in which the necessary physical constants and the behavior of different electrode materials were determined.

The purpose of the present paper is to give a general review of these investigations from the standpoint of the engineer who is concerned with the proper design and operation of furnaces, and to discuss more particularly the practical bearing of the results of the experimental part, including the proper propor- 
tioning of the electrodes, the selection of the best material, the calculation of the losses, the indications of the faults in existing constructions and their remedies, etc.

Some parts of these investigations have been published or are being published elsewhere, to which those interested are referred. The present paper will include only a general summary of these and will discuss more particularly the experimentally determined data not included in the other papers.

Fundamental principles. The fundamental principle of the present analysis of the electrode problem, is that the heat gradient at the hot end should be zero; that is, the line representing it should be horizontal at that end. This means that no heat will then traverse through the hot end, either one way or the other; hence no heat from the furnace or its products is lost through the electrode, and the product can therefore not be " chilled" by the electrode as has often been found to be the case with improperly proportioned electrodes. Such an electrode is a perfect heat insulator, better even than the walls.

This zero gradient can be obtained only by having the temperature of the hot end of the electrode equal to that of the furnace. In the present method this is done by so proportioning the electrode that the current through it will raise the temperature of the hot end to this furnace temperature.

Although the writer's first recommendation of this fundamental principle was met with scepticism and even ridicule, it is now believed to be generally accepted as the correct one. One's first impression, that this would consume much energy, is found to be incorrect; the explanation is briefly, that as the heat near the hot end has no easy means of escape, (for as it cannot get into the furnace it must all flow out at the cold terminal) it will rapidly accumulate, so that a small amount of energy will soon produce a high temperature. Under simplifying assumptions, (and approximately under all conditions*) this state of temperature equilibrium, is found to be also the condition of minimum total loss of energy in the electrode.

Although this way of operating an electrode is quite the contrary to that dictated by prior practice, which was based on the lowest practicable resistance, it turns out to be the most economical, even though it may involve an intentional increase of the resistance loss; it is the total loss which should be considered, as the watts of heat lost either from the furnace or in the electrodes cost the same.

*Metal. \& Chem. Engineering, Apri1, 1910, p. 188. 
Another advantage is that the whole interior of the furnace then becomes useful, because a furnace in which there was a chilling action around the electrodes, by a mere change of proportions of the electrodes, based on this principle, can then have its capacity increased to that of its full interior size.

It furthermore means that all the heat escaping at the cold end is then the $I^{2} R$ heat. Or inversely, if all the heat escaping at the cold end is the total $I^{2} R$ heat, and neither more or less, then the temperature of the inner end will be that of the furnace, and there will be no loss of furnace heat and therefore no chilling.

It is evident that this condition can be reached regardless of how good or bad a heat conductor the electrode material is; even with such a very good heat conductor as copper. Hence, even without any further analysis, this shows the fallacy of the oft repeated and generally accepted statement that a good heat conducting electrod: material necessarily chills the furnace, and is therefore objectionable. It will at once be seen that electrodes of good heat conducting materials must simply be made smaller in section than others.

Besides this fundamental principle there are a number of other features in which the writer's conclusions differ very radically from our former practice. The second one is in the determination of the total loss of energy in an electrode. According to the usual former practice, as shown in even very recent papers, the total loss was assumed to be the sum of that due to heat conduction alone and that generated in the electrode itself by the current. The writer found that this also was a fallacy and was not founded on a correct analysis. The true total is the sum of the conduction heat and only half the $I^{2} R$ heat, under the simplest condition of constant conductivities and no loss of heat to the walls. This has since been confirmed by others also and is now, it seems, generally accepted. Under the more complicated conditions of varying conductivities, it is claimed by some to be only approximately true, but in any case it is much more nearly correct than the older method.

It is easily seen why it should be so. The heat conducted from the furnace when there is no current, flows over the whole length of the electrode, hence the drop of temperature is proportional to the total flow; but the $I^{2} R$ heat is generated throughout the whole length, hence is equivalent to the whole of it entering at the middle and flowing over only half the length, or to half of it flowing over the whole length. Hence as far as 
the drop of temperature between the ends is concerned, only half of that corresponding to the $I^{2} R$ heat must be added to that due to conduction alone.

A third point of difference, and one which is still adhered to tenaciously by some, concerns the current density. This was formerly the basis of electrode design and is still considered so by some writers. The present investigation, however, has shown this to be a fallacy also. It has shown that the current density does not enter as a fundamental factor which determines the proportions. Even more than that; to base the proportions on current densities may even mislead one into using entirely incorrect proportions with unnecessarily large electrodes and losses of energy, accompanied by a false assurance that it is the best that can be done. It may have been found that when certain current densities were exceeded, troubles arose, but in the writer's opinion the mistake made was in attributing them to the current densities instead of to the length; it can be shown that the same current density will cause the electrode to become too hot or too cold, depending upon the length of the electrode. And conversely, for a given length a fixed current density prescribed by rule of thumb may be either much too high or much too low. Current density cannot therefore be a determining factor; it is no more a factor in the proportioning of electrodes than it is in calculating transmission lines, and should be abandoned the same as it was in the latter case years ago.

Since the writer pointed this out, a crude attempt has been made to defend the older practice by claiming rather vaguely that in some way the current density should be modified with the length. This is "beating around the bush" and would be an unnecessarily awkward and roundabout method, even if definite rules which are directly applicable had been given by the defender of that method, which was not the case.

The writer's conclusions are that current densities need not be considered at all as a determining factor, not any more so, and perhaps even less so, than in the calculation of transmission lines. $\mathrm{He}$ has operated electrodes very successfully and with the greatest possible economy of power at heretofore unheard-of current densities; and he knows of cases in which far lower current densities were concluded (erroneously) by furnace engineers to have been too high.

The fourth point of difference in the present method is in the resistance. The writer has found that the usual rule, to make 
it as low as practicable, is a mistake. It can be shown that the resistance for the most economical operation is not at all a matter of choice. It is determined by the conditions of the problem and is fixed by the temperature, current and material; it is different for different materials; hence to try to make the resistances the same for different materials is improper designing.

This investigation, of course, applies to the electrode proper, or what might be termed the essential electrode, or the chief part of an electrode, namely the part which passes through the walls, and in which the coopperation of the two heat flows is the governing feature. Any additional parts within the furnace or projecting beyond the outside of the walls, are not theoretically essential parts even though they may be very necessary in practice; such parts are evidently determined by entirely different conditions and considerations and must be treated apart from that portion which is absolutely necessary to lead the current from the outside to the inside of the furnace. To attempt to combine the various parts of such a longer electrode and to treat them as one in an analysis for finding out the laws governing the proportions, would either be impossible or would at least lead to endless complications rather than to a simplification.

Hence throughout the present paper the proportions of the electrode and particularly the length, refer to this essential part passing through the wall; the prolongations at either or both ends, if any, (as for instance the part embraced by the metallic terminal, or the extra part allowed for feeding) must be determined separately, being governed by entirely different considerations. When the metallic terminal surrounding the outside end is close against the wall and relatively short as compared with the part within the walls, it would probably be sufficient for most practical purposes to consider the electrode proper as ending at the middle of this terminal. And unless the cooling water is very close to the electrode, and the heat resistance between the electrode and ccoling water is very low, the temperature at the virtual end of the essential part of the electrode will be higher, and perhaps considerably higher, than the cooling water, hence the drop of temperature in the electrode proper will be less; it will be shown below that the hotter the outside terminal, the smaller the loss in the electrode.

This shows the fifth point of difference from our former views. Instead of trying to keep the outside terminals as cool as practicable, we ought to try so to design them as to let them get as 
hot as practicable, even to the extent of heating them with a blast lamp, when such heat is cheaper than electric heat. This will economize power, provided of course that the electric heat comes from the electrode proper and not from a poor terminal contact. The section of the electrode, however, becomes larger by raising the outside temperature.

A sixth point of difference from former practice which is brought out by this analysis is that instead of tending to make electrodes large, we should on the contrary try to make them small, as they can then be just as efficient; and there may even be other advantages also, besides economy of material, terminals, etc., in doing so.

Another complete departure from former methods is in the abandoning of the conductivities of the materials as factors in calculating electrodes and electrode losses. The writer found that the desirable qualities of electrodes do not depend on either the electric or the thermal conductivity alone, but on certain relations between the two. The analysis shows that our former deep-rooted conviction that a high heat conductivity is a bad feature for an electrode, is entirely wrong. The fact is, that it may be a good or a bad quality, depending upon the electrical conductivity. Neither alone is a criterion or a measure of excellence, and to consider them so misleads us.

In the writer's method of designing electrodes both qualities have been abandoned entirely as a basis of proportioning, and they have been replaced by two new qualities which are true and correct measures of excellence of those materials when they are used for electrode purposes; moreover they greatly simplify the calculations. Apparently the only objection to them is that they are not yet found in that form in tables of physical properties; they are however easily calculated from the conductivities, if the latter are known. That they are new and unfamiliar quantities will, it is believed, not be considered an objection by those who will take the time to understand their meaning.

These two new measures of electrode qualities are the "electrode voltage" and the "specific cross-section", these specific names having been given them in order to distinguish them clearly from other quantities. They are both measures of physical properties, quite as much so as conductivities, specific heats, specific gravities, etc., and are constants for the particular materials; that is, they are independent of 
the dimensions. The former concerns the power loss and the latter the size. The lower their values are the better is the material for electrodes. They will be further discussed below.

\section{ANALytical}

In an analytical investigation to determine the theoretical relations or laws in a complicated case involving many variable factors, especially in an entirely new field, the writer has followed, and is entirely in accord with, the teachings of such able authorities as Thomson (Lord Kelvin) and Taite, and doubtless of others also; namely, that it is best to solve such a problem first under the simplest possible conditions or premises, as a first approximation, and afterward to consider the refinements (if necessary) as a second or even third approximation. An attempt at the start to solve the complete problem with all its numerous less important refinements, as advocated by some of the writer's critics, would be more likely to obscure and lead us away from those simple and instructive approximate relations, which are often so valuable to the constructing engineer, than to lead us toward them and to point them out to us. A minor factor which might be absolutely negligible in practical calculations may give rise to very great complications in strictly correct algebraic expressions of a desired relation, thereby completely obscuring the more useful approximate one.

Hence in the present investigation, after a number of trials, it was found best first to eliminate all but the main or essential factors by limiting the premises to the simplest conditions. This made it possible to determine the fundamental relations or laws of electrodes, which prove to be very interesting and useful. By proceeding in this way, it was found that most, and perhaps all, of the more important corrections due to the minor factors, like the variations of the conductivities with temperature, could be combined into two experimentally determined coefficients or constants, and by the use of these in the original formulas, all the important, and perhaps even also the unimportant corrections can readily be included. A method was then devised by the writer for determining these constants experimentally, and was carried out by him; the results will be given below. This combination method is believed to be a simpler and more practical way of solving the complete problem with all its intricacies, than the purely analytical one, which even if it were completed, could not be used in practice until certain extended 
experimental determinations had been made, as no one has yet made them. As the present method of using the simplest possible formulas and embodying all the correction factors in two experimentally determined constants, seems to be not only simple but even more accurate and reliable than the other more complicated method would be, it is believed that the necessity for the other solution and for the long series of experimental determinations, which it is based upon, no longer exists.

The premises of the present simplified analysis are: that the cross-section is uniform; that the two conductivities have the same values over the whole length; that the electrode is heat insulated except at its ends; also that the Thomson effect, the skin effect, and other similar minor factors are neglected. Under these premises the following relations are rigid and exact, and may therefore be called the laws of electrode losses.

\section{Lawe of electrode losses:}

$a$. The combined loss through the cold end of an electrode is equivalent to the sum of the loss by heat conduction alone (when there is no current) and half the $I^{2} R$ loss.

$b$. This combined loss will be least when the loss by heat conduction alone is made equal to half the $I^{2} R$ loss; the total loss will then be equal to the $I^{2} R$ loss, and no heat will be conducted from the interior of the furnace.

c. This minimum loss is dependent only on the material, current and temperature, but not on the absolute dimensions; it merely fixes the relation of the cross-section to the length, but leaves a choice of either; hence

d. For economy of electrode material the length should be made as short as practical considerations permit.

$e$. For each material there is a definite minimum loss of electrode voltage which depends only on the temperature and is independent of the dimensions or the normal current for which. the furnace is designed; hence

$f$. The best possible electrode efficiency for any material may be determined from the total voltage of the furnace and this minimum voltage due to the material and the temperature, and is independent of the dimensions.

$g$. The temperatures indicated by the heat gradient of the combined flow are equal to the sums of those of the individual flows.

The proof of these laws is given in a paper by the writer on 
"Laws of Electrode Losses in Electric Furnaces",* and need not be repeated here.

The starting point is the fundamental principle, first announced by the writer a year ago as the proper one, namely, that no heat should leave or enter the furnace through the electrode or in other words, that the heat generated by the electrical resistance shall raise the temperature of the hot end to that of the furnace, it is shown that under the given conditions this is also the con dition of least total loss.

$W$ atts, a measure of flow of heat. Before giving the formulas the writer desires to explain that it is quite correct, and it simplifies such calculations greatly, to represent and measure a flow of heat in terms of the electric unit watts, instead of in calories per second. A watt is just as correct a measure of a flow or current of heat (calories per second), as an ampere is for measuring a flow of electric current in coulombs per second. Heat is energy, and a rate of flow of heat per second is power, hence it is measurable in units of power like watts. Since the writer called attention to this, others have endeavored to improve matters by calling this unit "watt seconds per second", but this cumbersome name is entirely unnecessary and obscures rather than simplifies our conceptions. It is evident that watts $\times$ seconds $\div$ seconds $=$ watts. When the heat conductivity of a material is given as 10 watts for an inch cube, it simply means that with one degree cent. difference of temperature between two parallel sides, and perfect heat insulation on the other four, the same amount of energy will flow through as heat, say into water at the cold end, as would enter the water from a coil of resistance wire in which 10 watts were being set free.

In the following, therefore, all flows of heat will be represented in watts, and it is recommended that in future all thermal constants pertaining to electrodes be given in terms of watts instead of calories per second. The conversion factors are: gram calories per second $\times 4.18617=$ watts, and watts $\times 0.238882=$ gram calories per second.

Formulas. The following formulas are the same whether inches or centimetres are used, provided they are employed consistently throughout, including all those constants which are based on dimensions, and which of course will be different; those like the electrode voltage or watts per ampere are, of course, the same in both systems. All the formulas in this paper are in

*Trans. Amer. Electrochem. Soc. Vol. 16, p. 265. 
terms of actual units and may therefore be used directly in practice. They refer to one electrode.

Let $S=$ cross-section in square inches;

$L=$ length in inches (the essential length);

$I=$ current in amperes;

$W=$ watts generated electrically in the electrode;

$H=$ heat flow in watts which would flow if there were no current;

$h=$ heat flow in watts which enters the hot end from the furnace;

$X=$ total heat flow in watts leaving the cold end;

$T=$ temperature drop in centigrade degrees between the hot and cold ends;

$r=$ electrical resistivity in ohm, inch cube units;

$k=$ thermal conductivity in watt, inch cube units;

$e=$ electrode voltage in volts;

$E=$ total voltage between the two ends, or the watts per ampere;

$s=$ specific cross-section in square inches;

$S^{\prime}=$ section in square inches per ampere per inch of length (or in sq. $\mathrm{cm}$. per ampere per $\mathrm{cm}$. length if the other quantities are in terms of centimetres).

In general, the flow of heat in watts at the cold end is

$$
X=H+W \div 2
$$

and entering at the hot end

$$
h=H-W \div 2
$$

in which

$$
H=k T S \div L
$$

and

$$
W=r I^{2} L \div S
$$

The total flow out of the cold end will be a minimum when

$$
H=W \div 2
$$


Representing this minimum flow by $m X$, then

$$
m X=2 H=W=I \sqrt{2 k r T}=I e \sqrt{T}
$$

this it will be seen does not contain either $S$ or $L$, which means that this is the same for all dimensions; it includes the condition however that the ratio of the section to the length is

$$
S \div L=I \sqrt{r \div 2 k T}
$$

which means that either the length or the section may be made anything one desires, provided only that the ratio is equal to the above. As the length is usually fixed by other conditions this formula is best written

$$
S=I L \sqrt{r \div 2 k T}
$$

The electrode voltage is

$$
e=\sqrt{2 k r}
$$

and the total voltage is

$$
E=\sqrt{2 k r T}=e \sqrt{T}
$$

both of which are seen to be independent of the dimensions or the current, the electrode voltage being even independent of everything except the properties of the material, and hence itself a physical constant. This means that for every material there is a fixed and definite voltage for one degree which voltage is the same no matter what the size of the electrode or the normal current, provided only that it is correctly proportioned so that no heat flows through the hot end.

Conversely, the total voltage $E$ being known, a convenient way is at hand of finding out whether the hot-end temperature of the electrode is that of the furnace or not, as this voltage might be measured without much difficulty during the operation of the furnace; if the measured voltage is found to be less, the electrode is chilling the furnace, if greater, the electrode is getting hotter within the wall than it is in the furnace.

The electrode voltage is that physical constant which is a true 
measure of the loss of power in an electrode. When multiplied by the square root of the temperature drop and by the current, as shown in (5), it gives the minimum loss in watts which can possibly be obtained with that material, for that current and temperature. Hence if its value is known, it is not necessary to know the two conductivities in order to calculate this loss of power.

The quantity $E$ is measured directly in the experimental determinations described later; hence it is known for each temperature and material. Substituting its value (9) in equation (5) gives

$$
m X=I E
$$

That is, the minimum loss in watts may be determined directly by multiplying this value of $E$ by the current; hence the voltage $E$ may be called the "watts per ampere".

This shows the simplified method suggested by the writer, for calculating these minimum losses. It consists in tabulating for each material the values of $E$ for different temperatures, as obtained directly by experiment (by the method described below); then for any given case, in which of course the current and temperature are given, one needs merely to multiply the corresponding value of $E$ by this current to get the result. This is so simple that it can often be done mentally. The values of the conductivities need therefore not be known.

Returning to formula (7), if $I, L$ and $T$ are made unity, that is, for 1 ampere, 1 in. length and 1 degree cent. of temperature, the resulting cross-section, now represented by $s$, becomes

$$
s=\sqrt{r \div 2 k}
$$

This the writer proposes to call the "specific section", because it is a physical property of the material, as its value, like that of the electrode voltage, depends only on the relations of other physical properties. It is a true measure of the size of an electrode, of course always under the condition that the electrode operates as was specified. This quantity therefore is the mate to the electrode voltage and determines the size just as the latter determines the loss.

Now let $S^{\prime}$ be another quantity so that

$$
S^{\prime}=s \div \sqrt{T}=\sqrt{r \div 2 k T}
$$


It will then be seen that in the same way and for the same purpose as was described above for $E$ as compared with $e$, it is possible to determine by direct measurement (by the method described below) the values of this quantity $S^{\prime}$ for various temperatures and for each material. When these values are tabulated it again becomes an extremely simple matter to calculate the proper cross-section, because

$$
S=I L S^{\prime}
$$

that is, one needs merely to multiply this value of $S^{\prime}$ from the tables by the current and the length to get the actual crosssection. This quantity $S^{\prime}$ is therefore here termed the " section per ampere per inch of length". Hence this calculation is also reduced to a surprisingly simple one, provided these tabulated values are at hand. Such a set of values of both $S^{\prime}$ and $E$ is given below in Table II.

When $S^{\prime}$ is not known, then

$$
S=I L s \div \sqrt{T}
$$

It will be seen that again the two conductivities drop out and need therefore not be known. In the absence of these tables the values of $e$ and $s$ should be used. If they too are unknown the mean conductivities must be resorted to. If in turn these electrode means are not known and the conductivities at the specified temperature are known, the mean values must first be determined from them. If these conductivities vary greatly with the temperature, a closer approximation could be obtained by taking into consideration the mutual effects of these variations on each other and on the mean, as described later in the discussion of the experimental results.

In this connection the writer desires again to call attention to the fact on which particular stress was laid in his first published description of his method, and which fact has been entirely ignored by the critics, that the curve of the combined losses is quite flat at its minimum point. This means that a considerable error can be made in the cross-section without affecting the power loss appreciably; for instance in a certain case an error of 10 to 20 per cent in the cross-section near the minimum point produced an error of only 1 to 3 per cent in the loss. Hence great accuracy in determining the values of these conductivities is 
not required for engineering purposes, even though to the physicist these slight variations produce great complications in the algebraic analysis. The degree of complication produced by a factor in a rigid mathematical analysis is no measure of its real importance.

Another reason why great accuracy in the values of these conductivities is not necessary for engineering purposes is that they both occur under the square root sign in the formulas used for electrodes (5) and (6); hence if one of them, as for instance the thermal conductivity which is the one least best known, is four times too great, the result will be only twice too great.

Various other relations exist between these old and new quantities which at times are of interest and use. They are easily deduced and are therefore merely summarized here.

$$
\begin{aligned}
& e=I \div W \sqrt{T} \\
& e=r \div s \\
& s=e \div 2 k
\end{aligned}
$$

Mean values and equivalent electrodes. All the above is based on the premise that the temperature variations of the physical constants may be neglected; this so greatly simplifies the formulas and relations that the numerous useful and interesting results given above, are obtained. They would certainly not have been brought to light from the extremely complicated relations which result when the temperature variations are introduced algebraically. This is the reason why the writer prefers the method of studying a new problem by means of simplified assumptions first, leaving the corrections due to small variations to be introduced afterward as a second or third approximation, if indeed such accuracy is necessary in calculations of furnaces which unlike dynamos, transformers and other electrical apparatus, cannot possibly be built or operated under very exact specifications. Hence great accuracy in the present investigation is merely of academic rather than of engineering interest.

In the complete form of the method as described by the writer in the original paper on this subject and repeated here, the variations of the conductivities are taken care of very effectively and no doubt more effectively than by intricate calculations, 
by determining the constants under the very same conditions under which they are to be used. This point was also overlooked by the critics. It embodies the desired correction factors in the constants themselves, hence they need no further attention. Even if the values at specific temperatures are known, which is not the case for carbon and graphite, the writer doubts very much whether the final results calculated from them by means of the more involved and decidedly more complicated algebraic relations, would prove to be as close to the actual as they are when determined by the present extremely simple method.

In the present method the values of the conductivities and other deduced factors obtained as will be described, are the means peculiar to electrode conditions. What they really represent are the values of another or equivalent electrode the conductivities and other properties of which are the same as these mean values, and are constant from end to end, that is, they are independent of the different temperatures along the electrode.

Such an equivalent electrode will as a whole operate in exactly the same way, and hence, as far as the flows of heat at the two ends are concerned, it is theoretically identical. It differs however in the shape of that part of the temperature gradient which is intermediate between these two end points. Hence, whenever this intermediate heat gradient is concern $\_d$ and only then, one must consider the nature of the temperature variations. This will be shown below in the discussion of the experimental results.

A brief summary of the discussion which has taken place concerning this and other features of the writer's method, will be given at the end of this paper.

\section{EXPERIMENTAL}

Most engineering calculations of structural work are based on the physical properties of materials often called "constants" even though their values vary somewhat; the proper designing of electrodes is no exception. When not done in this way it becomes a process of more or less skillful guessing which ought not to be called engineering. Until these constants are determined, calculations based on them of course cannot be made. At the time when the present investigation was begun some of the necessary physical proparties were either not known at all, or known only very vaguely and indefinitely; this refers chiefly 
to the heat conductivities. No particular need for them had existed, but since it had become possible to calculate electrodes on a rational basis, the need of these constants was felt. The writer therefore undertook a determination of them for graphite, carbon, iron and copper. The tests were made in the well equipped Electrical Testing Laboratories in New York City.

The method used was the one suggested in his recent paper on "A New Method of Measuring Mean Thermal and Electrical Conductivities of Furnace Electrodes."* It is based on the same fundamental principles and formulas as those of this analysis. Briefly it consists in effect in operating a pair of electrodes under conditions approximating as nearly as possible those under which they should (according to this analysis) be operated in practice, namely that the electrodes shall not chill the furnace; or more scientifically speaking, that the heat gradient at the furnace end shall be zero, represented by a horizontal temperature line, in which case there will be no flow of heat into or out of the furnace through the hot end of the electrode.

While thus operating, the current, voltage and cold temperatures are carefully measured for various furnace temperatures. The heat flow is by this method measured electrically in terms of watts before it is heat, thereby avoiding the necessarily cumbersome and often inaccurate measurements of flows of heat in the form of heat. From the data obtained the mean thermal and electrical conductivities or resistivities and several even more important constants are calculated by the formulas given, which are practically the same as those in which they are afterwards used to calculate other electrodes. - Further details are given in the above mentioned paper.

The method therefore amounts to measuring the constants of an electrode under actual operating conditions, and when these conditions are what they should be in practice. Hence it is very direct and does not involve any questionable process of determining the constants under one set of conditions and applying them to entirely different ones. The only important difference between the conditions of the test and those of the subsequent application of the constants, is in the dimensions of the electrodes. These constants are specific quantities; that is they are reduced to values per inch or centimetre units, hence they apply equally well to large or small electrodes, if minor effects, such as the differences in the ratio of the surface to the volume, are

*Trans. Amer. Electrochem. Soc. Vol. 16, p. 317. 
neglected. If further proof of this is necessary, it has been furnished by Dr. E. F. Northrup, who in an unpublished article has demonstrated that even under the complex conditions of the operation of electrodes, it is strictly correct to apply the constants obtained by this method to electrodes of different lengths or cross sections or both, provided that the temperature conditions at the ends are the same, and that the heat insulation is also relatively the same.*

The constants given by this test are the mean values for the electrode as a whole and when operating under the proper conditions. The conductivities thus obtained are not necessarily the arithmetic means of those at the ends or even the averages taken at equally spaced distances. They are means peculiar to operating electrodes, and their relations to the end values or to the mean of the values at equally spaced distances, are different for different materials. For this reason the writer has proposed the term "electrode mean " to designate them. They are the correct mean values to be used in the formulas determined by the present analysis, and as it would be difficult to calculate them accurately from values like those given in the usual tables, for different specified temperatures (if such values existed for electrode materials which they apparently do not), they are best determined experimentally by a direct method like the one described, if indeed it is not also an easier and more reliable method than those formerly used; it gives the proper mean values directly, while the other methods do not. While great accuracy in these constants is seldom if ever necessary in practice for calculating electrodes, it is desirable, to use them in an investigation like the present, in which comparisons and other deductions are to be made and in which the behavior of electrodes is to be studied.

The reasons why these mean values are peculiar to electrodes, will be best seen below.

This phase of the subject is discussed more in detail in an article published elsewhere. $\dagger$ It will be sufficient to say here that these means depend upon the distribution of the heat in the electrodes; that is, on the temperature curve. This is different for different materials and depends on the joint coöperation of both the electric and the thermal conductivities, because each affects the other. These means are in fact the values of an equivalent electrode which as a whole would operate exactly

*Trans. Amer. Electrochem. Soc. Vo1. 17, 1910, p 215 bottom.

$\dagger$ Metal. \& Chem. Engineering, March, 1910, p. 128. 
like the original one, as explained above. Hence in the present method these differences in the temperature curves are eliminated as far as the action of the electrode as a whole is concerned; they need be considered only in connection with the relations between the electrode and the surrounding furnace wall.

The details of the determination of these constants are described in a paper to be read before the American Electrochemical Society at its Spring meeting*. The purpose here is to compare and discuss the results, and to endeavor to point out what they teach us. It will be sufficient to say here that the tests were made with care; the results, as far as they go, are believed to be reliable and even more accurate than is required for most practical purposes. Sets of measurements were made for each of several temperatures for each material, and the final result of each run is an average of a number of very closely agreeing sets of readings. The furnace temperatures were kept constant for periods of about 10 to $30 \mathrm{~min}$. to within about 1 per cent and generally considerably less, it being an important feature of this test to reach the stable state of temperature.

The iron and copper electrodes were run to as near their melting points as practicable. The iron had unfortunately been injured slightly by an accidental excessive temperature prior to the last test, near its melting point, hence the values at the highest temperature are not as closely accurate and reliable as the others, but they are probably not far wrong. The same was the case with the highest temperature values for graphite. Allowances for these have been made in the curves. Less care was taken with the lower temperature values as they were of less importance.

Above about 900 degrees cent. the graphite and carbon electrodes began to be affected presumably by the gases in the granular insulating material, (magnesite), probably by the $\mathrm{CO}$ from the incompletely calcined carbonate. The results beyond that point were therefore thrown out, and those near it may not be quite correct.

The full lines in the accompanying curve sheets represent the ranges over which the results were actually measured; the dotted lines are exterpolated and therefore are only probable values. These exterpolated values were added here with some hesitancy as the writer is well aware of the possible danger in relying on the extensions of formulas and curves beyond the actual measured ranges. In the present case however the several curves for each

\footnotetext{
*Trans. Am. Electrochem. Soc., Vol. 17, 1910, p. 151.
} 
material are linked by fixed relations, hence it is not like simply prolonging individual curves; an incorrect extension of one curve will be likely to show on the others, and perhaps in an exaggerated form. For this reason the extension of each one aids in determining the extensions of some of the others. They are therefore probably more nearly correct than mere unguided and independent extensions would be; in fact it seems likely that these fixed relations are far better guides for these extensions than the curvatures of the known parts of individual curves.

In all these curves the exterpolated values were checked at 2000 degrees cent. and 1400 degrees cent. by these relations, and whatever differences were found were distributed over the curves where they corresponded best with the curvatures of the known parts. A second approximation was thus obtained and this was again adjusted, and so on until a final agreement was reached. This care was taken because at present there exist no reliable data concerning these mean values for those temperatures, and it was thought that the exterpolated values were at least better than none at all, as they were not likely to be far wrong. They are offered here merely for what they are worth and nothing more. The tests for determining these constants become tedious for the higher temperatures on account of the oxidation or other forms of deterioration of the carbon and graphite, and the melting of the metals. It may therefore be some time before the tests are repeated for those temperatures. Until then these exterpolated values may perhaps be of some service. Attention may here again be called to the fact pointed out above, that the curve of minimum loss is rather flat at the minimum point, hence quite a large error in the correct proportions will give rise to only a small one in the energy loss; close accuracy in the constants is therefore not necessary.

There may, of course, be radical changes in these physical constants at the melting points of the metals, and at some points at which carbon may change its condition. These cannot be predicted. The present extensions are all based on a regular continuation of the measured relations.

Although all the electrodes were very nearly $\frac{5}{8}$ in. in diameter they differed slightly, hence for comparing them more correctıy with each other, the results have here all been reduced to a diameter exactly of $\frac{5}{8}$ in. For the same reason they have also all been reduced to a constant cold terminal temperature of 
100 degrees cent. by adding or subtracting the required amount to both temperatures. The error involved in doing this is no doubt absolutely negligible for the present purposes. The data were obtained from electrodes 8 in. long measured between the furnace end and the furnace side of the water-cooled terminal.

When the method described in this paper is used for calculating electrodes, the important physical constants in these determinations are the electrode voltage and the specific section described above, and others related to them $\left(E\right.$ and $\left.S^{\prime}\right)$. The two conductivities are then eliminated as separate quantities and need not be considered. They have nevertheless been included in the following curve sheets as matters of interest and of use in studying the subject. The original measured data, namely the current and volts have also been added, after reducing the former as described; they lead to interesting and valuable deductions.

As the current densities in some of these runs were extraordinarily high, it may be of interest to add here that the electrodes behaved very well, so well in fact that the writer would not hesitate to use such extreme current densities in regular practice under the proper conditions. The results prove his contention, which has been disputed by others, that the current density is absolutely no factor in the determination of the proportions of electrodes. As the curves for the values of the current densities would be exactly like those for the curren', they have not been repeated on the curve-sheets.

Experimental data. The experimental results and the deductions calculated from the data are given in the accompanying Table I which explains itself; the exterpolated data are given in italics so as to distinguish them from the others.

In order to compare and study these data more readily, they have been plotted in Figs 1 to 11. In Figs. 1 to 4 the properties are compared for each of the materials, while in Figs. 5 to 9 the materials are compared under each of the properties. In Figs. 10 and 11 the two most important of these properties are compared; they were omitted from the other sheets for the sake of clearness.

In all the curves the scales give their actual values and not merely relative ones; hence as these scales are very different for the different materials, it is not proper to compare with each other the curves of the same property for the different materials without making due allowance for the different scales; they may be compared directly only as far as percentage differences ar? 
concerned. In the second group, Figs. 5 to 9 all the results of one kind are reduced to the same scales, and may therefore be correctly compared quantitatively.

All these data refer to electrodes when operating under the condition that the hot end is raised by the current to the exact furnace temperature, and when the electrode, therefore, does not abstract any heat from the furnace. The values of all the specific properties (resistivities, conductivities, electrode voltages and specific sections) are the means under electrode conditions for the electrode as a whole; that is, they are the values of an equivalent electrode which as a whole will operate like the original one, but in which these properties have the same values throughout its entire length. The values for the currents, watts and current densities apply of course only to the particular size of the electrode tested and will be different for electrodes of other dimensions. The voltages, however, apply also to any other sizes of electrodes and to any other currents, provided only that the electrode is properly proportioned and is operated under the normal specified conditions and with the normal current.

Besides the actual numerical values of the various quantities, the point of special interest in this set of curves is whether the specific properties (that is, the properties per unit conditions) are sufficiently near to constant to be assumed so in ordinary practice, and also whether they rise with the temperature or fall, as this makes a difference in their behavior.

Carbon. Fig. 1. The current curve rises rapidly and nearly in proportion to the temperature. This means that changes in the current will produce nearly proportionate changes in the hotend temperatures, hence variations in current affect the losses considerably, and a current which is considerably greater than the normal for which the electrode was designed, will tend greatly to overheat the electrode within the walls.

The watts increase nearly according to a diagonal line, as with all the other materials tested, except that for carbon there is a more pronounced deviation at low temperatures, which is also seen in some of the other curves, indicating that the properties of carbon follow somewhat different laws at the lower than at the higher temperature ; or perhaps, that at the higher temperatures, they follow laws similar to those which other materials follow at the lower ones.

The electrical resistivity diminishes, at first more rapidly, then less so, tending to become more nearly constant. A de- 
creasing resistivity means that as the current heats the electrode, the heat generated per inch will become less and less at the hot end and relatively greater at the coldend, than it was

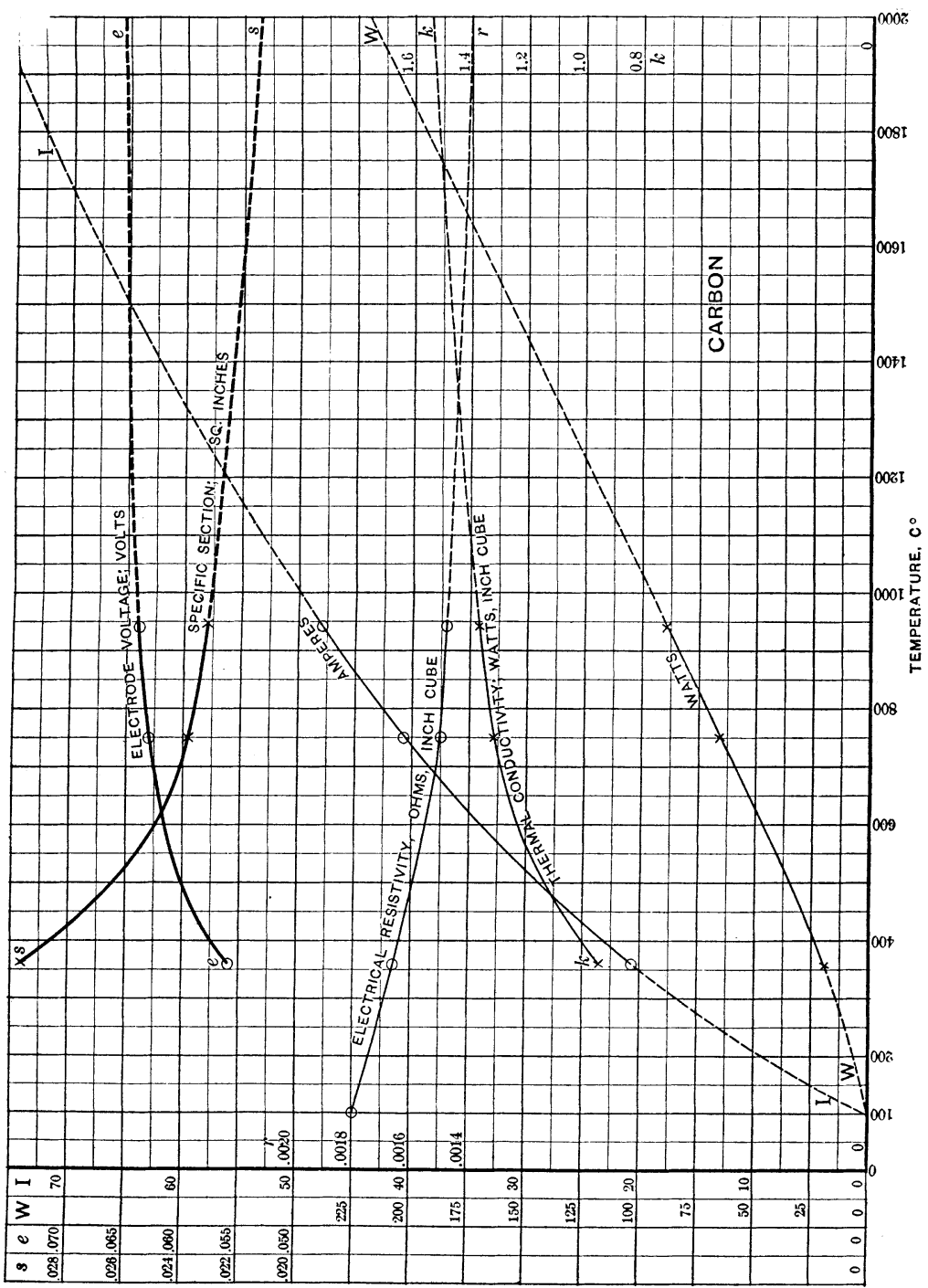

FIG. 1.-Electrode properties of carbon

before; hence the colder half will be heated more rapidly. This in turn signifies that the heat gradient will tend to approach a horizontal line from the hot end to near the cold one, and will 
then drop suddenly. Or in popular terms, the furnace heat will follow the electrode deeper into the walls. This seems to be one of the causes of the burning of the furnace wall in high-

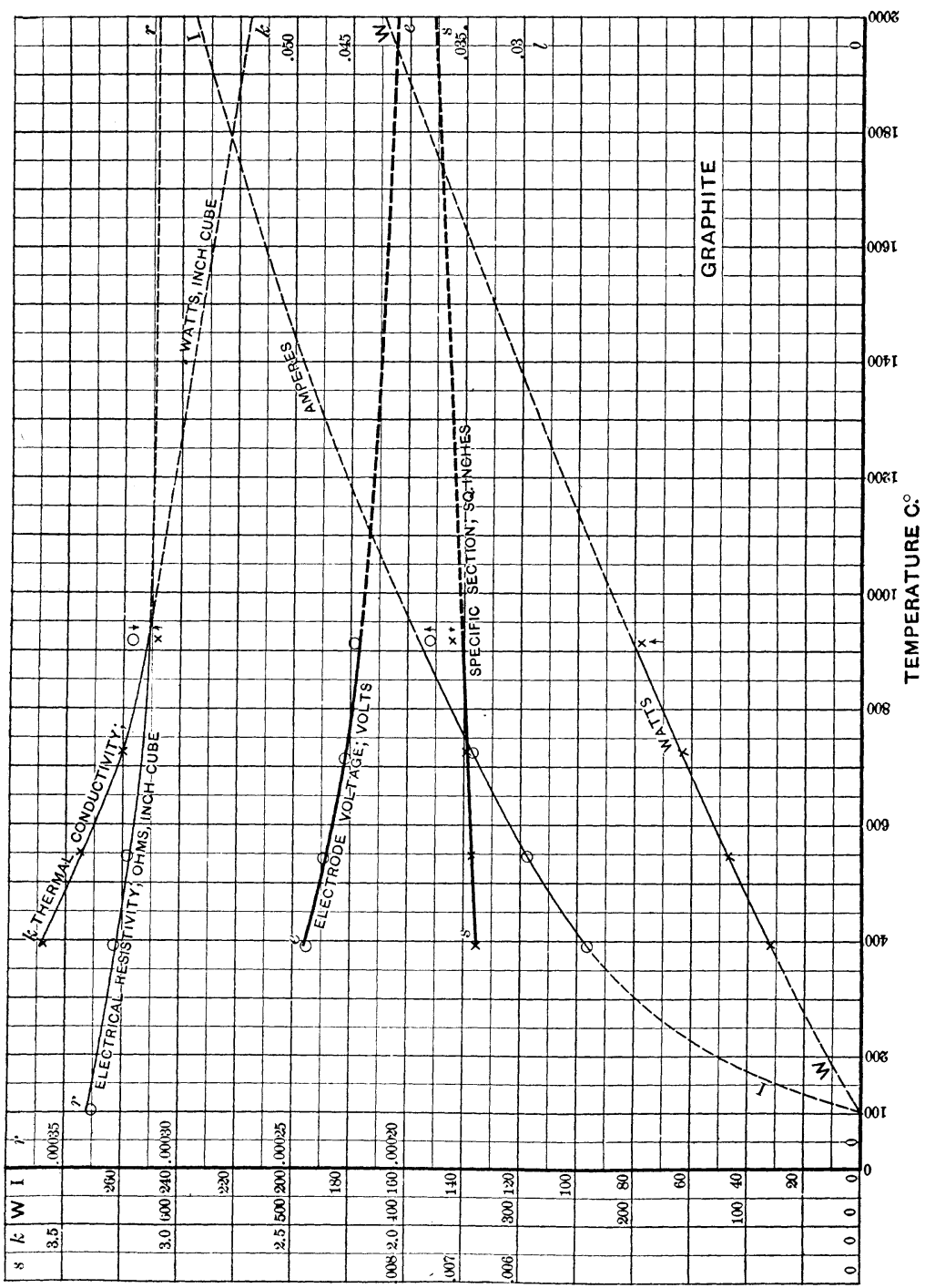

FIG. 2.--Electrode properties of graphite

temperature furnaces in which carbon electrodes are used. A falling resistivity is therefore an undesirable quality for electrodes. 
A rapidly rising one would be far better; this is the case with iron.

The thermal conductivity increases (it does not decrease as has been claimed by others) at first more rapidly, then less so, also tending to become more nearly constant. The surprisingly high values for the mean thermal conductivities are no doubt explained by the fact that the electrical resistivity falls quite appreciably with an increase of temperature. This in an electrode means that the larger half of the heat generated in the electrodes will be produced in the colder half, hence nearer the outlet, as explained above; it therefore has a shorter distance to travel in order to get out. The mean effective thermal conductivity calculated from the heat which flows out and from the dimensions of the electrode. will therefore be increased, and will even be larger than an average of that taken at equally spaced points. But the value obtained in these tests is the real one which is wanted, as it is the one which applies to the electrode as a whole; it is the value which an electrode of uniform conductivities would have, which, as a whole, would operate like this one. This unequal distribution of heat in electrodes will be discussed by the writer more in detail in an article to be published elsewhere.* The difficulty in calculating the mean from individual values lies in not knowing the heat distribution, that is, the temperature curve; also in the fact that these individual values for different temperatures have not yet been determined. The writer for these reasons recommended the present method as the more direct one by which to get the final results in the form in which they are wanted.

A rising thermal conductivity is an undesirable feature for electrode materials, as it tends to force the higher temperatures nearer to the colder end. Carbon unfortunately combines this feature with a falling resistivity, both of which conduce to the same undesirable result.

The curves for carbon furthermore show that the electrode voltage, which is a true measure of the power loss, also increases with the temperature, but the rate is slower and slower, tending to constancy. It will be shown later that there are reasons to believe that it may even reverse again and fall at still higher temperatures. The loss in carbon electrodes therefore increases not only with the temperature, but also per degree of temperature; at least for these ranges. The chief conclusion, as far as our present knowledge goes, is that probably no great error would

*Metal. \& Chem. Engineering, March 1910, p. 128. 
be made by assuming this important quality to be practically a constant, namely about 0.065 volt.

The specific section, which is a true measure of the size of an electrode, decreases rapidly at first and then more slowly, showing that carbon electrodes become relatively smaller for the higher temperatures; they are even smaller per degree at the higher temperatures. This is a good feature, and especially so for carbon because such electrodes are much larger than those of other materials, as will be seen later.

The electrode voltage and the specific section, are properties of the material, just as the specific resistance is, and if their values are constant or virtually so, calculations are simplified. The curves show that for most purposes in practice they may be assumed to be virtually constant for the higher values of this range of temperature.

The curves of the total voltages and of the actual size of the electrodes, being the ones which are the most important in practice, have for clearness been drawn apart from the others and will be discussed below in connection with Figs. 10 and 11.

The comparison of the quantitative values of the properties of carbon with those of other materials, is best made later in connection with Figs. 5 to 9.

Graphite. Fig. 2. The current curve rises more rapidly at first than for carbon, and in this respect graphite apparently possesses properties more like those of the metals. After this rise at the lower temperatures, it seems to approach more nearly to a straight line. The inclination of the latter part is less than for carbon, which indicates that for the same proportionate change in current the change of temperature produced thereby will be greater for graphite. From this it would appear to follow that a graphite electrode is more sensitive to changes of current than is one of carbon.

The curve of watts is practically a diagonal line, which shows that the watts lost in a given electrode will increase very nearly in proportion with the temperature drop, due to the current.

The mean electrical resistivity decreases slightly at first, and then seems to tend to constancy, at about 0.00031 in inch units $(0.00081 \mathrm{in} \mathrm{cm}$. units). It varies considerably less than for carbon, therefore the heat generated in the electrode will show a tendency to be more evenly distributed along the length, although the greater half of the $I^{2} R$ heat is as in carbon, generated in the colder half of the electrode. Hence graphite stands between carbon and the metals. 
A comparison of these mean values with those obtained by others when the whole rod is at the same temperature is of interest. It shows that these mean values are higher than the arithmetical means between the two extremes. This appears to be due to the fact that owing to a more rapidly decreasing thermal conductivity, the heat is forced back toward the hot end by the changes in thermal conductivity, more than it is forced to the cold end by the changes in the electrical conductivity. The result is that the average between the extreme temperatures would be greater than the actual; hence the mean resistivity found in these tests should be greater, which is the fact, if these data are correct. This places graphite among the metals in this desirable quality of forcing the heat back to the hot end.

The curve for the thermal conductivity shows that it falls as has already been stated. In this feature graphite differs decidedly from carbon (for which this curve rises) and is like the metals. The thermal conductivity falls more rapidly at first and then displays a tendency to become more nearly constant. Its rather high value is due partly to the falling electrical resistivity, as was explained above for carbon. It will be seen below that this brings its mean value even above that of iron, which has a rapidly rising resistivity.

The electrode voltage falls slightly, but seems to tend to what for all practical purposes may be assumed to be constant at about 0.040 to 0.045 volts. As this is a measure of the loss, it follows that the loss per degree diminishes slightly with the temperature instead of increasing, as it does with carbon. Graphite, therefore, is relatively to the temperature, more economical of power at higher temperatures than it is at the lower ones. This is due to the somewhat rapidly falling thermal conductivity.

The specific section is practically a horizontal line, and consequently may be assumed to be constant at about 0.0070 in square inches. This means that the size of the electrode relatively to the temperature is practically the same at high and at low temperatures; it does not (lecraase per degree, as with carbon, but even at the higher temperatures it is still much smaller. These comparisons are best seen below in other sets of curves.

Iron. Fig. 3. The current curve shows a marked peculiarity in that it is practically a horizontal line, differing radically in this respect from carbon and graphite. This is due nodoubt 
to the very rapidly rising $r$ sistivity. As the hot end becomes hotter the resistance rises so rapidly that it cuts down the current again. Such an electrode is therefore nearly self-regulating for a constant current. A slight increase of current causes a very great rise in temperature. This fact was not fully realized during the progress of the test, in which the current was at one time raised too high, which apparently melted or perhaps even volatilized the hot end; this occurred before the run at the highest reading, hence the values obtained for the last point are not as good as the others, and were given less weight. After the test bar had been removed it was found that it had been melted at the hottest point and had even partly disappeared. This must have happened before the last run was taken, as that was still below the melting temperature. Anyone who repeats this test should be very careful to increase the current but very little at a time, and should not try to hasten the time of reaching the steady state by a temporary excessive current.

This peculiar property of iron, to change greatly in temperature with slight increases of current, also interfered with the intention to avoid the region of the recalescent point, about 700 degrees cent., as it was to be expected that the electrical and thermal conductivities might also take part in the peculiar physical pranks which iron plays at that temperature. It is doubtless due to this recalescent point that the curves are not as regular as are those of the other materials. They have a noticeable hump at about 700 degrees cent. But notwithstanding these disturbing factors, the general trend of the curves enables instructive results to be deduced.

In the exterpolated (dotted) parts of these curves, it has been assumed that the laws of variations continue to be about the same. It is not unlikely, however, that with materials which melt, like metals, as distinguished from carbon and graphite, there may be decided changes of these physical properties at the melting point. This can be determined only by experiment, and tests with molten columns the cross-section of which must remain constant, become difficult. Moreover, unless they were made on a large scale they would be seriously interfered with by the so-called "pinch effect", which would break the column unless it was very securely confined. Under the circumstances the exterpolated values may be of at least temporary use until someone has made the actual tests. But it should be remembered that they are nothing more than exterpolations. 
The curve of watts is as usual virtually a diagonal

Th e electrical resistivity rises greatly, and roughly in a straight line with only an indication that the rate probably falls at higher

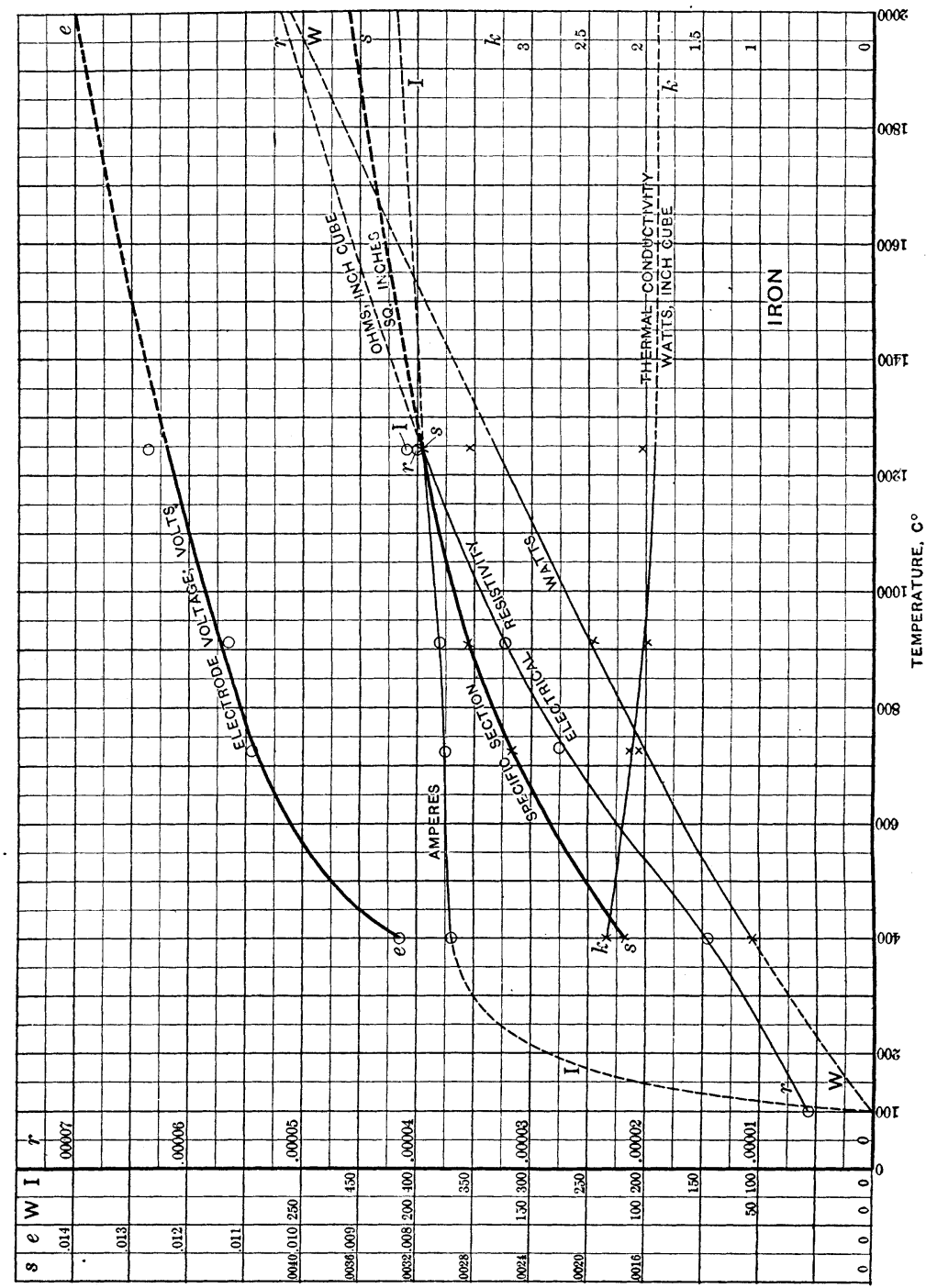

FIG. 3.-Electrode properties of iron

temperatures. This rapidly increasing resistivity is a very desirable feature for electrodes, as it forces the heat back to the hot end, as was explained above. 
The thermal conductivity falls, which is also a desirable feature. It is of interest to note that for iron both conductivities vary in the desirable way, while for graphite only one does (the other

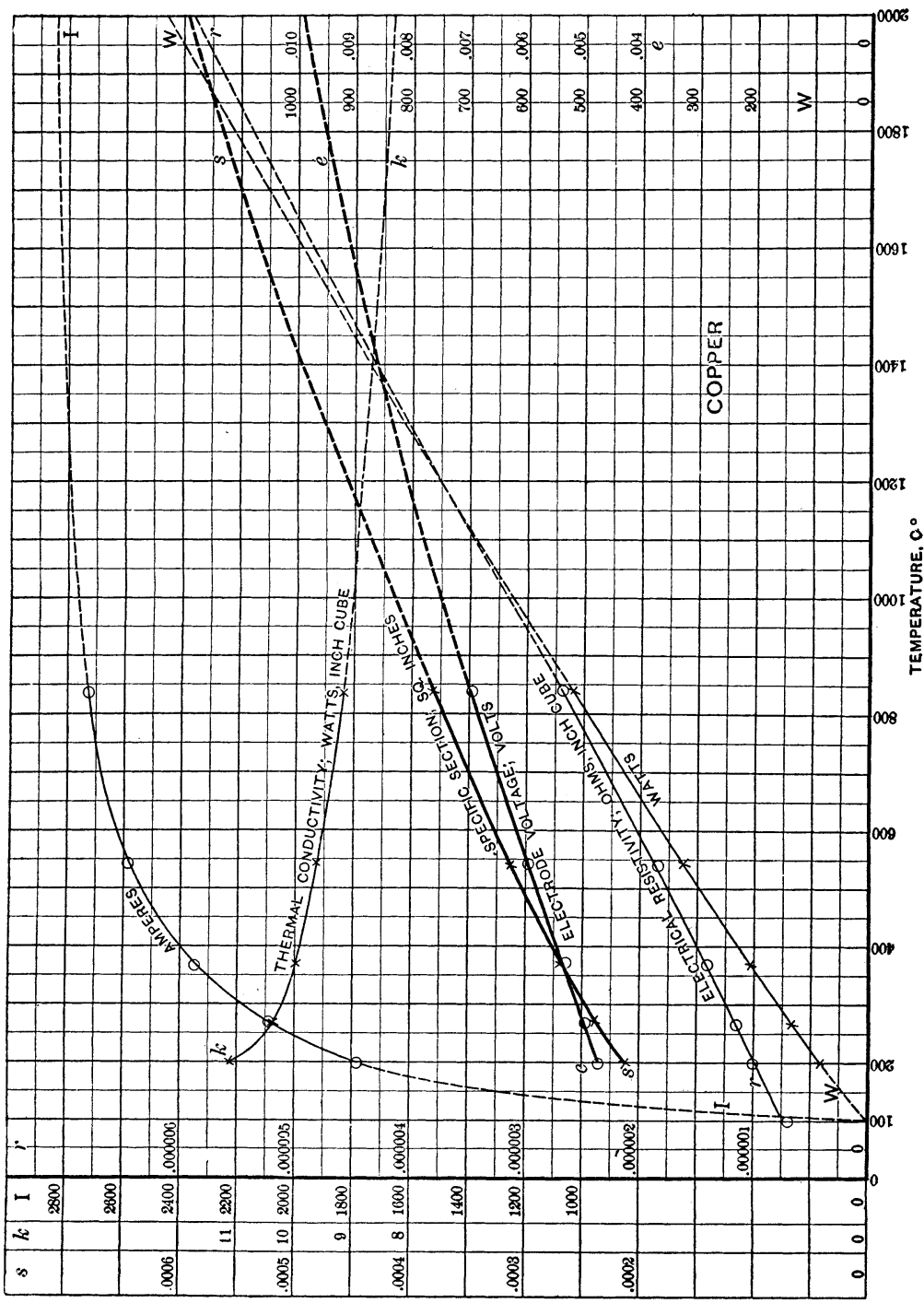

FIG. 4.-Electrode properties of copper

however being nearly constant), and for carbon, they both vary in the undesirable way. Although the thermal conductivity of iron falls, the amount is but slight and it may be assumed 
to be practically constant at about 1.9 to 2.0 in watts, inch cube units.

It will be noticed that the mean thermal conductivity is less than that of graphite. This may not agree with comparisons of the usual constants at specified temperatures; the explanation of the difference unquestionably lies in the fact that the heat is forced to the hot end more in iron than in graphite, as has been explained. The greater part of the heat having to travel a greater distance to get out, an iron electrode is rendered equivalent to one having a lower conductivity than the iron has at those temperatures.

Owing to this combined falling conductivity and rising resistivity, it seems that the temperature curve for iron must fall quite rapidly at the hot end and then approach the horizontal for the greater part of the length. This confines the high temperatures to the end near the furnace where they belong. It moreover has a tendency to make the temperature gradient in the electrode more like that in the walls, which signifies that little or no heat passes from one to the other. But one of the most important results is that when there is an excessive current, which tends to heat the electrode within the walls to a higher temperature than that of the furnace, this high temperature point will be very near the furnace, far nearer than it would be for carbon, for instance.

The electrode voltage rises quite decidedly. This means that iron electrodes are relatively less economical in power at the higher than at the lower temperatures; there are no indications of a constancy of the value of this property within these ranges.

The specific section also rises at about the same rate, showing that the size also becomes less favorable at the higher temperatures.

The test bars were cold rolled, mild steel.

Copper. Fig. 4. The tests with the copper electrodes were the best of the series. This was due partly to the fact that the currents, being very large, were better adapted to the output of the dynamo; partly to the fact that copper had a thermal conductivity so very much better than that of the surrounding heat insulating material, that the proportion of the loss to the surroundings was least; and lastly, because it was the last test of the set and the writer was enabled to embody the experience gained in testing the other materials.

The current curve rises extremely rapidly at first and then 
apparently tends rapidly toward the horizontal; it seems to be similar in shape to the one for iron at lower temperatures. It therefore appears that this shape may perhaps be characteristic of the metals. The last point was very near the melting point. hence the exterpolated values are based on the possibly incorrect assumption that no radical changes take place in these properties at the melting point.

The curve of watts is again practically a diagonal.

The electrical resistivity rises very rapidly and in virtually a straight line; copper resembles iron in this property, and the resistivity rises even slightly more rapidly in percentage. What was said about iron in this respect applies therefore also to copper. The heat will again be forced to the hot end.

The thermal conductivity also falls, as in the case of iron, only to a greater degree. This and the rising resistivity should make copper a very suitable electrode material for the temperatures for which it could be used.

The electrode voltage rises in almost a straight line proportion. So does the specific section. It is therefore like iron in these respects.

Comparisons. In all the above curve sheets the scales were chosen as large as the space permitted, hence they are all different. In order to compare the different materials with each other quantitatively all the curves for each property have been redrawn to uniform scales in Figs. 5-9.

Current. Fig. 5. These curves explain themselves. They show that at about 1500 degrees cent. a graphite electrode will carry over 3 times as much current as will one of carbon of the same size; iron will carry over twice as much as graphite or nearly seven times as much as carbon; and copper is enormously better than any of the others, its current capacity being about 45 times that of carbon or 14 times that of graphite. With what losses these electrodes carry these currents will appear later.

The curves also show a tendency to become horizontal. The actual numerical values refer to $\frac{5}{8}$-in. round electrodes and to no others, although relatively the currents would always have the same ratios.

Current densities. The cross-sections in all the test rods being the same, these curves also represent the relative values of the current densities. The actual values are given in Table I. Some of them will be seen to be exceedingly high. 


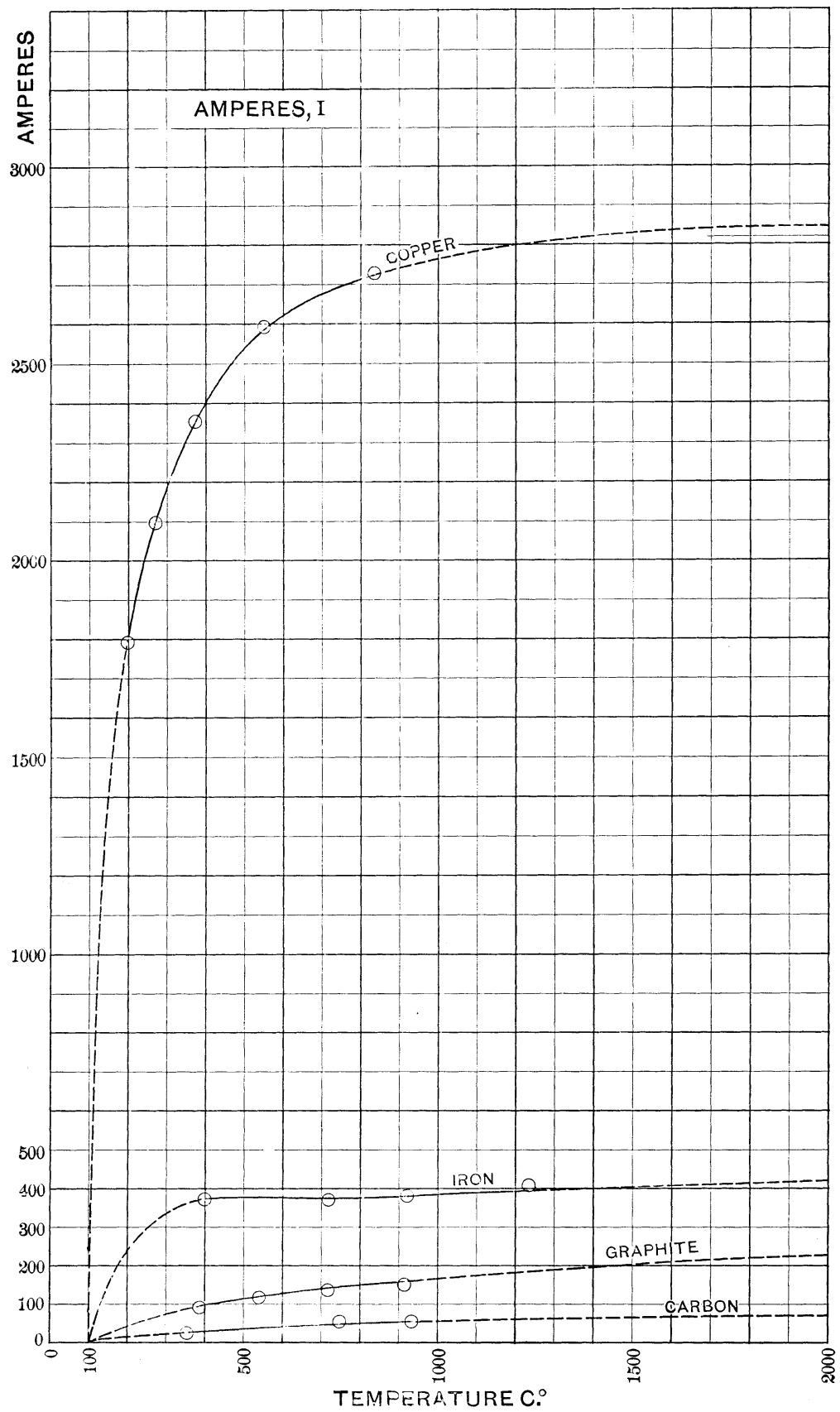

Fig. 5.-Current for different materials 
Watts. Fig. 6. The curves for all four materials are seen to be nearly diagonal lines, indicating that the watts increase nearly in proportion to the temperature required. In order to show how little they differ from the true diagonals, the latter have been added as thin lines to the last points. The difference is greatest with carbon. It can be shown that in so far as the real curve differs from the exact diagonal, in so far does the thermal conductivity vary from a horizontal line, that is, from constancy; if these curves approach the diagonal more nearly for the higher temperatures, as they seem to, it means that the thermal conductivities approach constancy for those higher temperatures.

Moreover if the bend is above the diagonal as it is for copper, graphite and iron, it signifies that the thermal conductivity falls, while if below, as for carbon, it rises. Furthermore, it can be shown that the thermal conductivity is proportional to the tangent of the angle which the diagonal to any point makes with the horizontal.

Further than this, the curves should not be compared, as they might mislead. Quantitatively they refer only to those particular electrodes $\frac{5}{8}$ in. in diameter, and to no other size. While the watts lost for copper are very much greater than for carbon, the current delivered was also far greater, and the relative loss was far less than for carbon. This is shown better in the later curves.

Electrical resistivity. Fig. 7. These curves show strikingly the quantitative relations, it being nearly impossible to show copper and carbon on the same sheet. A low electrical resistivity is a desirable quality in electrodes, both in economy of power and in the economy of materials. It shows why copper is so very much better than carbon, when it is possible to use it. Iron is near copper, and graphite is much nearer the metals than carbon. A rising resistivity is a good feature while a falling one is a bad one.

An interesting point is that the line for carbon falls rapidly, that for graphite also falls but very slightly, while those for the metals rise. Their relative inclinations make it appear as if they tended to meet at some very high temperature in a common point somewhat below graphite, at which point they would all have the same resistivity. At 1400 degre $\lrcorner$ s cent., the relative values are about: copper 1, iron 10, graphite 72 , carbon, 340 .

Thermal conductivity. Fig. 8. The thermal conductivities differ far less than the electrical resistivities; moreover the usual order is here reversed, graphite being now next to copper. They 
again all seem to tend to meet in a point, this time iron being the more central one. The relative values at 1400 degrees cent. are about: copper 1 , graphite 0.34 , iron 0.22 , carbon 0.17 . It may be repeated here that a falling thermal conductivity is a good feature and a rising one is a bad one; also that a high thermal conductivity reduces the size of an electrode but increases the loss; hence it is a good or a bad quality depending upon which of the two economies one desires. There are other properties which are better measures of excellence of an electrode material; they will be discussed below.

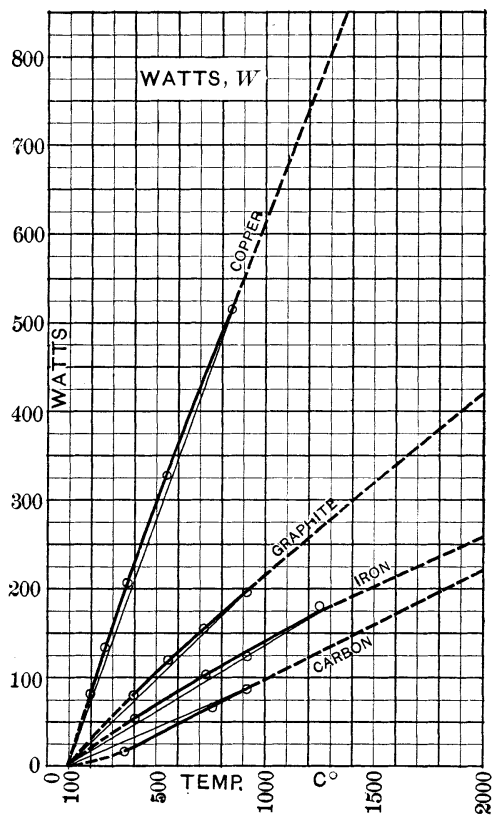

Fig. 6.-Watts for different materials

Electrode voltage. Fig. 9. This is the specific property which is a true measure of the minimum watts lost in an electrode, when it is properly proportioned. Its significance has been described above.

The curves are shown in thin lines. They all show a tendency to rise except for graphite which alone possesses the good property of a falling curve. As all these specific quantities seem to tend to meet at some very high temperature, it may be that carbon which is the only exception, will also fall again at higher temperatures, as indicated. 
Iron is now nearly as good as copper and much better than graphite, although the difference seems to grow less at higher temperatures. The lines for graphite and carbon depart at first, but then remain nearly parallel. The relative values at about 1400 degrees, are about: copper 1, iron 1.5, graphite 5, carbon 7.5.

Specific section. Fig. 9. This is the specific property which is a true measure of the size of an electrode, when properly proportioned. Its significance has been described above. The

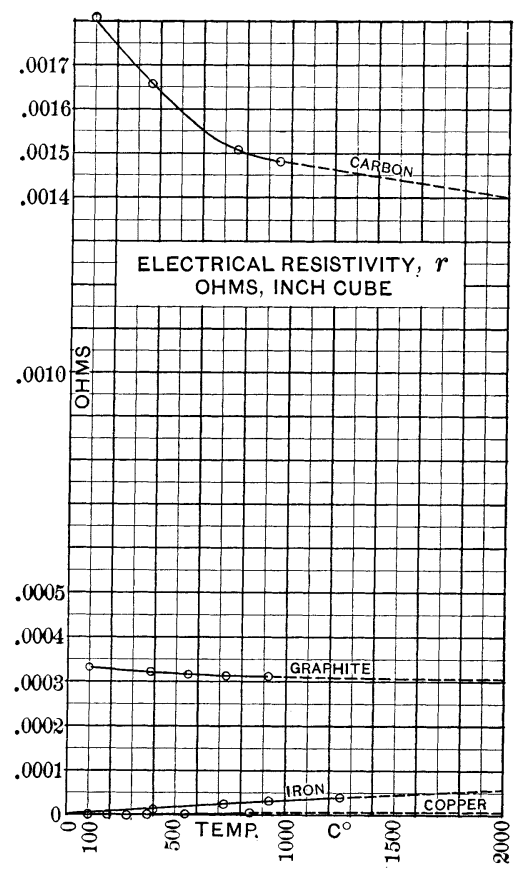

FIg. 7.-Mean electrical resistivities for different materials

curves are shown in heavy lines. They all tend to rise except for carbon; and again they seem to tend to meet. Copper as usual makes the best showing, and graphite is nearer the metals in this respect than it was for the electrode voltage. Carbon is far from the others, showing that such electrodes must be made very much larger. For about 1400 degrees cent. the relative values are about: copper 1, iron 6.6, graphite 14.6 , carbon 45 .

These two qualities have been drawn on the same sheet to facilitate comparisons. Carbon electrodes are seen to be both 
large and wasteful of power, those of graphite are much smaller and while they consume less power, the difference is not as great as that in the sizes. Copper is best in both respects, and iron comes next in both qualities, being also better in both than graphite, particularly in the economy of power.

Actual losses and sizes. Figs. 10 and 11. The electrode voltage and the specific section are the two physical constants which the writer suggests using instead of those formerly employed. Like most other physical constants they vary somewhat with the temperature, though some of them only very slightly; but they nevertheless belong correctly under the heading of physical

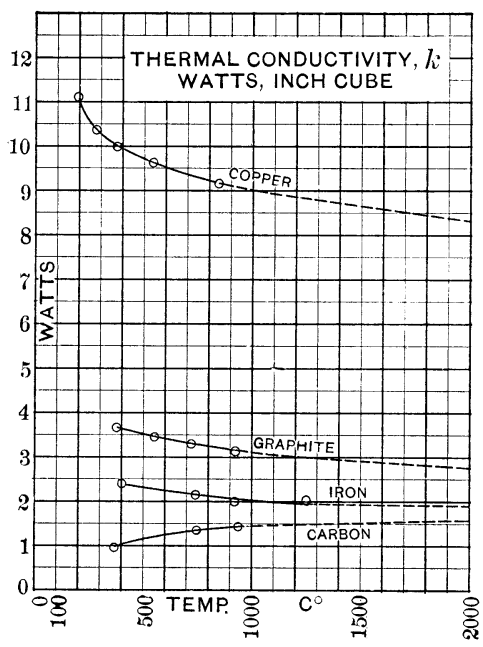

FIG. 8.-Mean thermal conductivities for different materials

constants, which can for many calculations be assumed to be constant, or have the differences allowed for, just as is done with resistivities in ordinary electrical conductors.

But as was explained above, the usually required calculations may be considerably simplified and reduced to a mere simple multiplication, by using instead a tabulated set of numbers. These are no longer "constants" in the usual sense of the term, because from their very nature they must have greatly different values for different temperature ranges, although for some materials and ranges the curves representing them are very nearly straight lines.

If, therefore, the designer has such a table of values at hand, 
he will no doubt prefer those values to using the physical constants. These two sets of figures give the watts per ampere, and the square inches of section per ampere per inch of length, for each temperature. Hence to find the minimum loss in watts for any given material and temperature (the cold terminal temperature being 100 degrees cent.) one merely multiplies the corresponding number of "watts per ampere" from the table, by the current in amperes, while to find the proper section in square inches it is necessary only to multiply the corresponding "section per ampere per inch length " by the current and the length

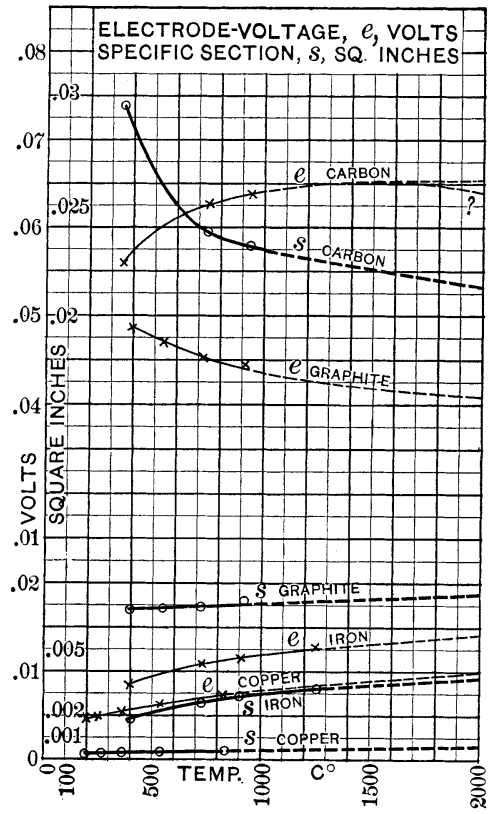

FIG. 9.--Electrode voltages and specific sections

in inches. The calculations are so simple that they may in many cases be made mentally. These values are given in Table II and are shown graphically in Figs. 10 and 11.

As shown above, the watts per ampere are in fact merely the voltages (in volts) between the two ends of an electrode when operating under the condition in which there is no loss of furnace heat. Hence these are not deduced figures, but are actually measured during the test, (being half the measured voltages of the two electrodes), while the figures for the sections per ampere 


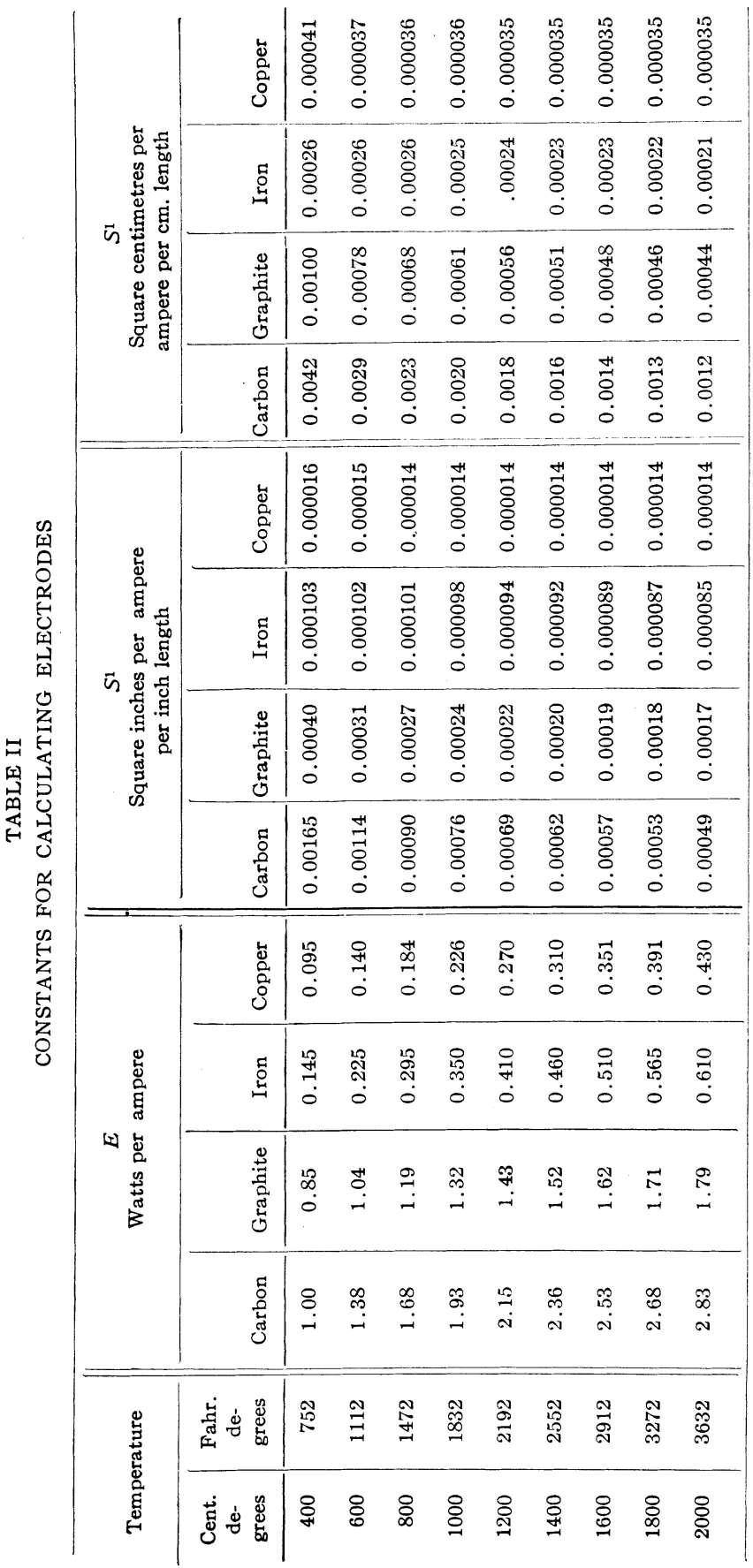


per inch length are merely the cross sections of the test rods divided by the length of the electrode (half the length of the double ones used in the test) and by the current.

It will be seen therefore that it is not at all necessary to determine or even to consider either the electrical or the thermal conductivity in electrode problems, if these simple data are at hand. And the writer's conclusions therefore are, that as little or no reliable data of any kind exists for the higher temperatures, and must therefore be ascertained, it is much simpler to determine and use these two new factors, instead of determining and using the mean conductivities. It is also infinitely simpler than the former method of determining the conductivities at each of various temperatures and then by means of more or less complicated and approximate formulas calculating the final results, with the uncertainty involved in all approximate algebraic deductions in which we do not always know how much the originally small, allowed errors may be magnified during the algebraic processes.

The writer's present method goes directly from the experimental result to the desired application in practice, without involving any assumptions other than that the relative distribution of the heat in the electrode for any given material and end temperatures, is the same for all cross-sections and lengths, (which is believed to be exactly correct under the assumption of no heat loss to the walls), and that the relative heat 1 )ss to the walls, whatever it may be, is approximately independent of the lengths and sections, (which is known to be not exactly true but probably sufficiently so for all practical purposes).

It should be noticed that no assumption is made that the heat loss to the walls is the same for all materials; on the contrary it will be different, perhaps appreciably so because, as was seen above, some materials (like carbon) have the property of forcing the high temperature toward the cold end, while others (like iron) force it back to the hot end. For this reason the temperature gradients will be quite different in the two cases, and the loss to the walls, if it is large enough to be considered at all, will therefore be different for different materials; but in the present method this difference is taken care of in the tabular data, and needs no further consideration.

The curves for these two final quantities are given apart from the others in Figs. 10 and 11. In Fig. 10 they have been drawn to different scales so that they are nearly superimposed, thus 
enabling their relative inclinations or percentage values to be compared better with each other, while in Fig. 11 they have all been drawn to the same scale, so that they may be compared quantitatively.

In Fig. 10 it will be seen that the curves $E$, which measure the minimum loss, or the watts per ampere, all have a family resemblance, except in the case of graphite; in fact with slight changes in the scales, those for iron, copper and carbon may nearly coincide, except at their lower temperature values, and this difference may be due to the experimental determinations which were not carried out quite as carefully at the lower temperatures for carbon and iron, as for copper. Even graphite might perhaps be brought into line by a change in the horizontal scale, which would mean that the temperature would have to be multiplied by a coefficient, or have a different exponent.

This family resemblance, and the fact that all these curves must have a zero and an infinite value, of course suggest the possibility of a common formula for all, differing only in its coefficients. But this is beyond the purpose of the present paper; the results obtained in this interesting direction will be made the subject of another discussion.*

The marked difference of the graphite curve means that the losses in graphite electrodes do not grow as rapidly with the temperature as they do for all the other materials. This, of course, is a very good quality, especially for high temperature furnaces. For all the others the relative increase of this loss is about the same. It is of interest to note that copper and carbon, the two extremes in all other comparisons, are here very nearly alike in their percentage variations.

A comparison of these same curves $E$ in Fig. 11, (the heavy line curves) shows the quantitative relations of the actual losses per ampere. The much greater loss for carbon is quite striking; but of course there are many cases in which metals are excluded by the very nature of the furnace. Consequently, tempting as it may be, the good quality of the metals cannot be taken advantage of. The relative values at 1400 degrees cent. are about: copper 1 , iron 1.5 , graphite 5 , carbon 7.5 ; the same, of course, as for the electrode voltage.

The loss for carbon is seen to be 1.55 times that for graphite. This is interesting in view of the claims that have been made that graphite is more wasteful of power than is carbon, even ten times as bad. Hence either the experimental data on which that

*Trans. Am. Electrochem. Soc., Vol. 17, p. 171. 
result was based were in error, or the method of deducing the result from them was incorrect; or else the curves change very radically at the higher temperatures, which seems hardly likely. If the present exterpolations are correct, this difference in favor of graphite becomes even greater at the higher temperatures.

Returning to Fig. 10, the thin line curves $S^{\prime}$ (the section per ampere and per inch) will also be seen to have a family resemblance, and as they also must have values for zero (100 degrees in this case) and infinity, the possibility of a general equation differing only in coefficients or exponents, suggests itself for this relation also.

If equations can be deduced for both $S^{\prime}$ and $E$, it will be possible to calculate both the conductivities from them, as one is proportional to the product, and the other to the quotient, of the conductivities. The coefficients for $S^{\prime}$ and $E$ are, of course, determined experimentally, as in the present case, and the conductivities will therefore be functions of these coefficients.

The exception in the $S^{\prime}$ curves is iron, but in general the two metals are similar to each other, as are the two non-metals, the two pairs differing somewhat.

Comparing them quantitatively in Fig. 11, the family resemblance is again apparent, the curves being evidently asymptotic to both ordinates, care being taken to deduct the 100 degrees cent. for the cold terminal temperature.

Carbon electrodes are again shown to be far larger than the others. The relative values at 1400 degrees are about: copper 1 , iron 6.6 , graphite 14.6 , carbon 45 , the same as for the specific sections. In this feature graphite is more like the metals and differs more from carbon than in the losses. The section for carbon will be about 3 times that for graphite. This difference in size, however, seems to diminish for higher temperatures, if the present exterpolations are correct.

Comparing both these important factors, it will be seen that graphite is better than carbon in both loss and size, especially in size. Copper is best in both and carbon worse in both, while iron is closer to copper in both than it is to graphite.

This final comparison is shown in a practical way in Fig. 12, in which the sizes and losses in electrodes of the different materials are drawn to scale for a given case, in which the current is assumed to be 10,000 amperes and the end temperatures 1400 degrees cent. and 100 degrees cent.

In the first set, to the left, the length is made the same for all, 
namely $10 \mathrm{in.}$, hence the sections will be different, and in the proportions of $1,6.6,14.5$, and 45 , the same as above given. The actual sizes in square inches are: copper 1.38, iron 9.16,

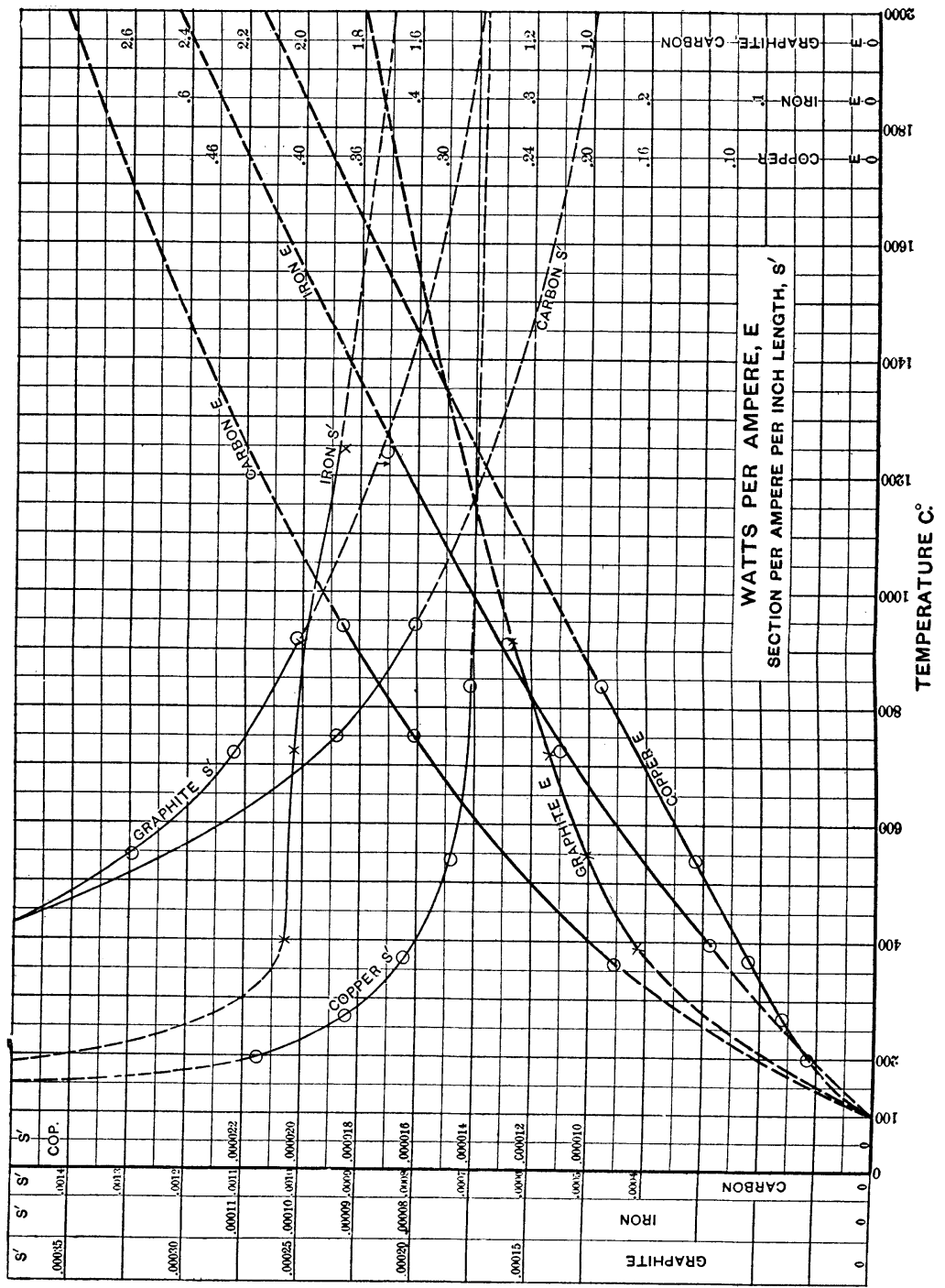

FIG. 10.-Losses and sizes; superimposed

graphite 20.1, carbon 62 . It will be seen that the proportions for carbon are practically impossible. 


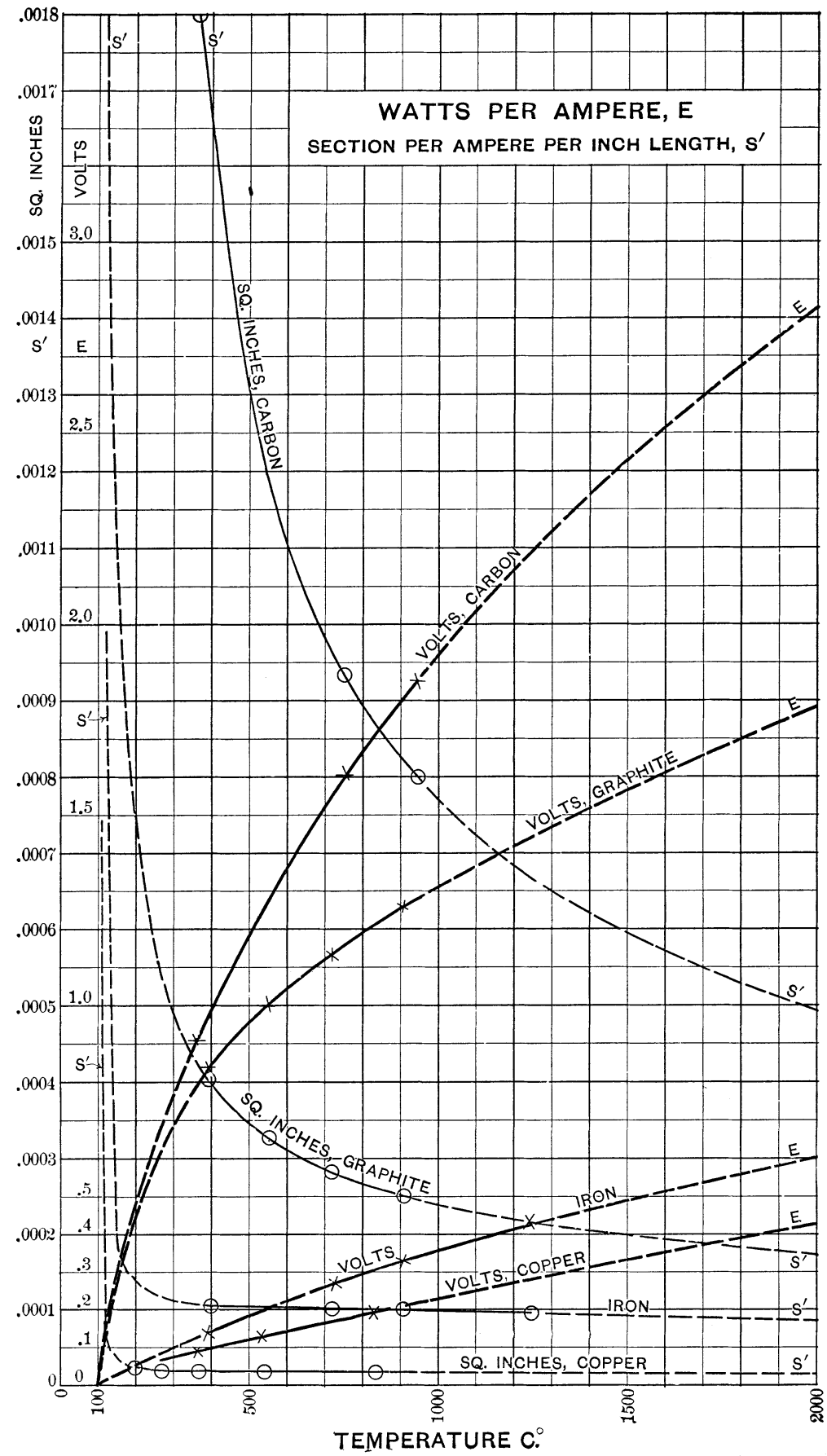

Fig. 11.-Losses and sizes; samer scale 
In the second set, to the right, the cross-section is made the same, the lengths being then different. They are in inches: $10,1.51,0.69,0.22$, dividing which by 10 gives their relative. values based on copper. That for carbon is again impossible, as are also those for graphite, and perhaps iron.

The losses in kilowatts per electrode are indicated by the lengths of the heavy black lines, which of course apply equally well to either of the two sizes for the same materials. They are in kilowatts, about: $23: 6,15.2,4.6$ and 3.1 , or in the ratio of $7.5,5.0,1.5$ and 1.

When the ratio of the section to the length is a constant, as in this case, the length increases more rapidly than the diameter. Hence to give the carbon electrode a suitable shape for handling, it must be made still larger, and considerably so. In comparing practical electrodes this feature should be considered, and it

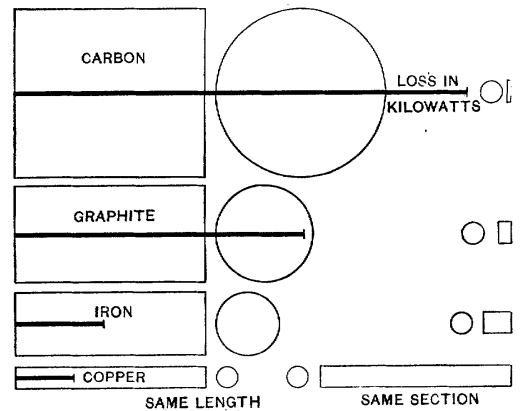

FIG. 12.-Comparison of sizes and losses

will increase still more the difference between the sizes of the carbon and the others. When finally the volumes are figured out, the differences will be found to be very great. But even when thus correctly proportioned the losses will still be much greater for carbon, as they are the same for all sizes, provided the relation of the section to the length is the same.

It is evidently not proper, as has been claimed, to lengthen the carbon electrode of the section shown, so as to give it a better shape, and to think that this merely means a small sacrifice in efficiency. Such a lengthening will evidently result in developing a high temperature within the walls, higher and perhaps much higher even than the furnace temperature. This would be likely to burn out the furnace wall, which would be fatal to successful operation. If it is made smaller and shorter 
the proportions will be still worse, as is seen in the upper right hand illustration. Consequently there seems nothing left to do but to make it much larger and longer.

This brings out another wrong deduction which has been made by others, based on the former incorrect rules. Suppose the carbon electrode in this Fig. 12 had been made with the same section but 20 in. long; it is evident from the results of the present analysis that a high temperature point, higher than that of the furnace, and probably very much so, would develop at a point somewhere between the furnace, and a point 10 in. from the cold end, because $10 \mathrm{in}$. is the correct length for that section. This would burn out the walls. The conclusion drawn from our former ideas was that the current density was too high, when the fact is that the electrode was too long for that section. The current density had nothing to do with the failure, because if the electrode had been made half as long instead of as long again, it would have been too cold and would have chilled the furnace, although the current densities were the same. Furthermore if the electrode had been made half the section and length shown in Fig. 12, it would have operated properly even though the current density had thereby been doubled. The current density, therefore, was not the cause of failure; it has often been made responsible for faults which were really due entirely to errors in the length.

\section{SUMmaRY}

The final results of this rather lengthy investigation are briefly as follows, as far as they interest the designer.

The underlying feature is that the electrode shall not chill the furnace, nor develop a high temperature point within the walls at which the temperature is greater than in the furnace. Hence the hot-end temperature should be as nearly as practicable equal to that of the furnace.

The current and furnace temperature are always given in the specifications. The material and either the length or the section (but not both) may also be specified, but preferably all three should be left to the designer. The voltage and loss cannot be specified, as they are determined by the current, temperature and material.

The length (that is, the essential length, not including additions to either or both ends for other purposes than to get the energy through the walls) may be determined solely by the thickness of the furnace walls; but as this affects the cross-section, 
some latitude should be left in case the corresponding section is found to be too large of too small to be practicable.

The section in square inches is then determined at once by multiplying the proper temperature value of $S^{\prime}$ from Table II by the current in amperes and by the length in inches (formula 13). If this is too large or too small to be practicable, the length may be changed and the section may be re-determined. The quotient of the section divided by the length is a constant for any specified conditions, (numerically equal to $S^{\prime}$ from the table multiplied by the current) hence they may both be increased or decreased in the same proportion.

Should this table of values not be available and the specific section be known, formula (14) should be used for calculating the section. If this is also not known, then use the conductivities in formula (7). Great accuracy is not necessary when one is near the correct result, as an error in section near that point produces a relatively much smaller error in the loss.

The loss in the electrode in watts is entirely independent of the section or length adopted, provided only that their quotient is approximately as above. It is calculated in watts by multiplying the corresponding temperature value of $E$ in Table II by the current (formula 10). If this table is not available, we may use the electrode voltage in the last part of formula (5). If this is not available either, then use the conductivities in the preceding expression of formula (5). This loss is for one electrode and it is the least possible under those conditions.

When the current for a furnace varies appreciably, the calculations must of course, be made for some assumed normal value, remembering that whenever it is less than that, the electrode will chill the furnace more or less; and whenever it is greater the alectrode will get hotter within the walls, and in both cases the total loss will be greater.

In operating a furnace, if the electrode is found to chill the product, it is either too short, or too large in section. If on the other hand it is found to produce excessive temperatures within the walls, it is too long or of too small section. The current density is not a determining factor.

The hotter the outside terminal is allowed to get, the smaller the loss, but the larger the section or shorter the electrode.

When the proportions turn out to be large in section and short in length (as for instance with carbon for large currents as in Fig. 12) the relative proportions may be improved by increasing 
both length and section. On the other hand, if the electrode is abnormally long and small in section (as for instance with iron or copper and small currents) then if the section cannot be made smaller, there seems to be nothing left to do except to sacrifice some of the loss by making the electrode shorter and it will then chill the furnace more or less.

The additional lengths of the electrode necessary for the terminals, for feeding, or for the distribution of the current in the inside, must be calculated separately, as they are determined by entirely different laws and conditions; the above refers only to the essential part which is necessary to get the current into and out of the furnace as well as possible. The above length must therefore never include the long external part outside the furnace, for feeding purposes. Such a part radiates heat to the air and therefore follows entirely different laws of proportions. A redeeming feature of such a case is that the outside temperature used to determine the proportions of the essential part, may be allowed to be very high, probably limited chiefly by oxidation thereby reducing the total loss which has been increased by the long external part.

Summary of previous discussions. The description of this new way of attacking the problem of the proportioning of electrodes, given by the writer briefly in May, 1909, and more in detail in October 1909, has given rise to long discussions and attacks. Some of the purely technical parts were of interest and value and will be summarzied briefly below. The rest of the discussion is of no general interest as it consisted of the usual unsubstantiated claims of priority made by those who suddenly found that they had known it long ago but had not given their knowledge to others or used it themselves. It contained also the assertions of those who claimed, after the method was described, that it was obvious though this had not occurred to them before; also the statements of those who maintained that on account of some academic minutia it was obviously fundamentally incorrect, although they could not substantiate their claims with actual figures; and the assertions of others who thought that the results were of no value because the necessary physical properties of the materials were then not yet known; etc.

One of the parts of the discussions which was of value referred to the magnitude of one of the corrections to which reference was made in the original paper, due to the simplified premises. This correction referred to the effect of the variation of the 
conductivities with temperature, and therefore is in the nature of a refinement or second approximation. Another important part was an ingenious graphical method devised by Dr. A. E. Kennelly,* for representing and studying the relations of the quantities involved.

Concerning the temperature correction, Dr. Kennelly has shown in a very able papert that under certain approximate premises, this correction is zero when the electrical resistivity varies according to a straight line law (the thermal one being constant). The correct mean value then is the arithmetic mean between the two extreme values at the two end temperatures. Also that the same is true when the thermal resistivity alone varies in this way. Also that when both vary in this way, the total loss is least when half of the heat flow at the cold end is joulean heat and the other half is conducted from the furnace; the remainder of the joulean heat is assumed to flow toward the furnace, thereby tending approximately to balance the furnace flow, hence a "very little heat will flow into or out of the furnace." This means that the condition of no loss of furnace heat under those approximate premises, is only a close approximation to the condition of minimum loss, instead of being identical, as it is with constant conductivities.

Dr. E. F. Roeber $\ddagger$ has attempted the complicated mathematical solution of the case when both conductivities vary according to a straight line law, and has carried it up to, but not including the integration. Although this unfinished formula is probably too complicated for use in practice by engineers, it shows that for given materials at stated temperatures the correction factor is merely the numerical coefficient, and that the relations between the variable factors remain the same. Dr. Roeber also finds that when the electrical conductivity is constant and the thermal conductivity alone varies by a straight line law, this complicated formula reduces to the simple original one in the writer's paper. This indicates that it is largely the variation of the electrical conductivity which gives rise to a correction

*Trans. Am. Electrochem. Soc., Vol. 16, p. 297.

†A paper " On the Modifications in Hering's Laws of Furnace Electrodes Introduced by Including Variations in Electric and Thermal Resistivities" published elsewhere in this volume.

f " Electrode Losses in Electric Furnaces," Trans. Am. Electrochem. Soc. Vol. 16, p. 363. See formula (5) p. 367. 
factor, a fact which the writer has since shown in another paper in a different way.

Dr. H. C. Richards* gives a very interesting solution of the integration for the complete case when both conductivities vary by straight line laws, and gives the first two terms of the series of coefficients. This enables the correction factors to be determined, when the conductivities and their variation with temperature are known. By means of this interesting solution it was shown by the writer $\dagger$ that even for very great variations in the conductivities, the corrections on which some of the critics based their unsubstantiated claims that the writer's approximate formulas were very incorrect and even "fundamentally wrong ", were in fact quite negligible in practice.

The writer takes this opportunity to express his appreciation of these able mathematical solutions by Dr. Kennelly, Dr. Richards, and Dr. Roeber, which show that differences between the writer's simple first approximation of the complete problem and the second approximation, are not great, and in part do not exist at all. Their work was done before the present experimental determinations had been undertaken, and before some of the simplifications described in the present paper had been made.

Those who, on the other hand, attempted to belittle and discredit the results of this investigation, brought up minor points which were either based on an incomplete reading of the original paper and are answered in the present paper, or were based on academic points which it is believed the practical engineer can safely neglect or allow for, as he does in most other construction work. The present investigation was made from the standpoint of the engineer and not from that of the academician or the mathematical physicist whose enlargement of negligible minutia is apt to obscure the main practical issues; nevertheless the analytical part of this investigation is undoubtedly rigidly exact under the specified simple conditions.

It is suggested that critics who endeavor to tear down and destroy the work of others by hastily made and unsubstantiated assertions, which may prove later to have been unwarranted or incorrect, might do some thing which is really of value, by devoting their efforts to improvements and further developments instead of to mere destruction. Before tearing down a structure built by others, one should be very sure first that it is

\footnotetext{
* Trans. Am. Electrochem. Soc. Vol. 16, p. 304.
}

† Ibid, p. 310. 
a dangerous one; if one thinks he can build a better one, then let both stand; the fittest will survive in the end without the need of the hand of the destroyer.

As to the usual crop of claimants of priority after something has been disclosed, the practice of secreting information of benefit to fellow engineers, until someone else has taken the trouble to publish it, and then of claiming priority and expecting recognition, is not in accordance with a high standard of professional ethics, and is believed to be more apt to discredit the claimant than to do him credit. Unpublished ideas are of no benefit to the profession at large; it is the one who takes the trouble to publish them, that aids his colleagues. 